\title{
Malnutrición: impacto de los hábitos, variables constitutivas y condiciones socioeconómicas en la población adulta urbana en la Argentina'
}

Malnutrition: Impact of Habits, Individual Factors and Living Conditions in Adult Urban Population in

Argentina

Subnutrição: impacto dos hábitos, variáveis constitutivos e condiç̦êes socioeconómicas na população adulta

urbana na Argentina

Karina Temporelli' ${ }^{2}$ Valentina Viego ${ }^{3}$

Recibido: 21 de abril de $2014 \bullet$ Aceptado: 17 de marzo de 2015

Doi: dx.doi.org/10.12804/revsalud13.02.2015.03

Para citar este artículo: Temporelli K, Viego V. Malnutrición: impacto de los hábitos, variables constitutivas y condiciones socioeconómicas en la población adulta urbana en la Argentina. Rev Cienc Salud. 2015;13(2):159-170. doi: dx.doi.org/10.12804/revsalud13.02.2015.03

\section{Resumen}

Introducción: el término malnutrición se refiere a los desequilibrios en la ingesta de energía, proteínas y/o nutrientes involucrados, tanto en la desnutrición como en la obesidad. El objetivo del trabajo es identificar el efecto de las variables constitutivas del sujeto, sus hábitos y condiciones socioeconómicas sobre categorías nutricionales de la población adulta urbana de la Argentina. Materiales y métodos: se estima un modelo logístico multinomial, aplicado a los datos de la Encuesta Nacional de Factores de Riesgo de 2005 y 2009. Se presentan, además, indicadores de riesgo relativo asociados con cada cofactor. Resultados: La desnutrición se asocia con las variables constitutivas y socioeconómicas, mientras que en la obesidad intervienen además los hábitos. Discusión: La complejidad de los problemas nutricionales en la Argentina, originada en la heterogeneidad en hábitos alimentarios y condiciones de vida, torna complejas las intervenciones necesarias para solucionarlos, destacando el carácter multidimensional de la problemática (médica, social, económica).

Palabras clave: Desnutrición, obesidad, hábitos, condiciones socioeconómicas.

1 Una versión preliminar del artículo ha sido expuesta en la Reunión Anual de la Asociación Argentina de Economía Política, que se realizó en la ciudad de Rosario, Argentina, los días 13, 14 y 15 de noviembre de 2013.

2 IIESS, Departamento Economía, Universidad Nacional del Sur. Correspondencia: 12 de Octubre y San Juan (8000) Bahía Blanca, Argentina. Correo electrónico: Ktempo@criba.edu.ar

3 Departamento Economía, Universidad Nacional del Sur. Correo electrónico: vviego@criba.edu.ar 


\begin{abstract}
Introduction: Malnutrition refers to imbalances (either deficit or excess) in energy intake, protein and/or nutrients, including underweight and obesity. The aim of this work is to identify the contribution of individual factors, habits and life conditions in nutritional status. Material and methods: Estimates of relative risk ratios are obtained from a multinomial logistic model using microdata from the National Survey of Risk Factors conducted in 2005 and 2009 among adult population in Argentinean cities. Results: Malnutrition is associated with constitutive and socioeconomic variables, while habits are also important in obesity cases. Discussion: Both types of problems coexist in Argentina due to a high heterogeneity in life styles, turning it difficult to design public interventions aimed to solve them. This complexity highlights the importance of a careful and specific multidimensional approach (medical, social, economic).
\end{abstract}

Key words: Malnutrition, obesity, habits, socioeconomic condition.

\title{
Resumo
}

Introdução: o termo subnutrição refere-se aos desequilíbrios na ingestão de energia, proteínas, e/ou nutrientes involucrados tanto na subnutrição como na obesidade. O objetivo do trabalho é identificar o efeito das variáveis constitutivas do sujeito, seus hábitos e condições socioeconómicas sobre categorias nutricionais da população adulta urbana da Argentina. Materiais e métodos: estima-se um modelo logístico multinomial aplicado aos dados da Enquete Nacional de Fatores de Risco de 2005 e 2009. Apresentam-se além indicadores de risco relativo associados a cada cofator. Resultados: a subnutrição se associa com as variáveis constitutivas e socioeconómicas, enquanto que na obesidade intervém além os hábitos. Discussão: a complexidade dos problemas nutricionais na Argentina, originada na heterogeneidade em hábitos alimentares e condições de vida, torna complexas as intervenções necessárias para solucioná-los destacando o carácter multidimensional da problemática (médica, social, económica).

Palavras-chaves: Subnutrição, obesidade, hábitos, condições socioeconómicas.

\section{Introducción}

El término malnutrición se refiere a las carencias, excesos o desequilibrios en la ingesta de energía, proteínas y/o nutrientes. Aunque el uso habitual del término no suele tenerlo en cuenta, su significado incluye tanto la desnutrición como la sobrealimentación.

Durante el siglo xx, las modificaciones en las conductas humanas, asociadas con cambios tecnológicos y la progresiva expansión del ingreso medio, dieron lugar a la llamada "revolución nutricional postindustrial", con cambios en los estilos de vida y aumento sostenido en el peso promedio de los individuos (1). Este fenómeno ha sumado a la obesidad al listado de problemas nutricionales aún no resueltos.

En los países de ingresos bajos, el hambre y la desnutrición siguen presentes conjuntamente con las enfermedades infecciosas y altas tasas de mortalidad infantil, mientras que en los países de ingresos altos han aumentado la prevalencia de individuos con sobrepeso y obesidad, patologías relacionadas estrechamente con las enfermedades crónicas no transmisibles. Por otro lado, en los países de ingresos medios ambas problemáticas se superponen (2). 
La relación entre las condiciones socioeconómicas y las patologías asociadas con la malnutrición es más evidente en el caso de desnutrición, donde los bajos ingresos y los déficits educativos pueden generar trampas de pobreza con limitaciones perdurables en el acceso a los alimentos tanto en cantidad como en calidad (3).

Si bien, no existe un consenso en cuanto a la relación entre las condiciones socioeconómicas y la obesidad, ya que el género, la edad o el origen étnico operan como cofactores de peso, en general, la literatura sugiere que en los países industrializados la prevalencia de obesidad es más significativa en los grupos de bajos ingresos, mientras que en los países en desarrollo la incidencia de la obesidad es mayor en los grupos de mayores ingresos (4-10).

El nivel de ingresos influye sobre el peso de los individuos, por medio de su efecto sobre el consumo de alimentos y gasto energético. Por un lado, el incremento de ingreso puede provocar un aumento del gasto en alimentos ricos en calorías, consumidos tanto dentro como fuera del hogar. Por otro, puede afectar la asignación del tiempo entre trabajo y recreación, si las calorías gastadas en el trabajo difieren de las gastadas en actividades extralaborales, el peso tiende paulatinamente a modificarse (11).

Además, la influencia de los factores socioeconómicos puede diferir, según la magnitud de esta problemática en cada país. En países con baja prevalencia de sobrepeso, aumentos del nivel de ingreso favorecen el tránsito hacia hábitos obesogénicos, mientras que en países con elevadas tasas de obesidad, un mayor nivel de ingresos puede atenuar el problema (12).

En la Argentina conviven ambos tipos de problemas, debido a una notable heterogeneidad en las condiciones socioeconómicas, lo que torna más complejas las intervenciones necesarias para solucionarlos y destaca la necesidad de un abordaje profundo de la problemática.

Una mirada hacia el interior del país nos enfrenta con algunas regiones con indicadores demográficos similares a la de los países desarrollados, tal el caso de la Ciudad Autónoma de Buenos Aires (CABA) y otras donde la transición demográfica aún no se ha completado. En los territorios, donde ello ha generado un efecto acumulativo, emergen disparidades epidemiológicas; coexisten enfermedades propias de un país desarrollado (enfermedades cardiovasculares, tumores, drogadicción, etcétera.), junto con enfermedades propias de países en desarrollo (enfermedades transmisibles o infecciosas) (13). En el campo socioeconómico, las diferencias regionales son marcadas, persisten la pobreza y la desigualdad en los ingresos, que se traducen inevitablemente en condiciones socioeconómicas poco satisfactorias y situaciones sanitarias deficitarias. Inevitablemente, estas disparidades llevan también a diferencias en las características nutricionales de la población y los problemas que de esto se derivan.

La tabla 1 presenta sintéticamente el alcance de la problemática nutricional en adultos de la Argentina, lo que muestra una cantidad creciente de adultos obesos (18\% en el 2009)

Tabla 1. Prevalencia de problemas nutricionales para la población urbana de la Argentina, 2005-2009

\begin{tabular}{ccccccc} 
& \multicolumn{2}{c}{ Desnutrición } & \multicolumn{2}{c}{ Sobrepeso } & \multicolumn{2}{c}{ Obesidad } \\
\cline { 2 - 6 } 2005 & $\%$ & Individuos & $\%$ & Individuos & $\%$ & Individuos \\
\cline { 2 - 6 } 2009 & 2,9 & 605000 & 31,6 & 7200000 & 14,6 & 3082236 \\
\hline
\end{tabular}

Fuente: realización propia en base a ENFR 2005 y RNFR 2009. 
e importante cantidad de adultos desnutridos (2,4\% en el 2009).

El objetivo del trabajo es identificar la contribución de las variables constitutivas, de hábitos y socioeconómicas, del individuo en las condiciones nutricionales de la población urbana adulta de la Argentina, utilizando los datos de la Encuesta Nacional de Factores de Riesgo (ENFR), mediante la estimación de un modelo logístico multinomial.

El análisis se focaliza en la población urbana, tanto por las características demográficas de la Argentina, donde, según datos del censo de población del año 2010, el 89,3 \% de la población reside en centros urbanos, como por la ausencia de microdatos para áreas rurales.

\section{Materiales y método}

La fuente de los datos empleados es la ENFR, realizada periódicamente por el Ministerio de Salud de la Nación. La ENFR publica indicadores de factores de riesgo de la población urbana de dieciocho años y más, que residen en localidades con más de 5000 habitantes. La información se obtiene de una muestra probabilística estratificada y multietápica aplicada a individuos en sus domicilios. A la fecha de elaboración del manuscrito, solo se accedía a los microdatos de los relevamientos realizados en 2005 y 2009, los correspondientes al año 2012 se encontraban todavía en procesamiento.

Con el fin de estimar los efectos de las variables constitutivas, hábitos y socioeconómicas, sobre los problemas de malnutrición de la población urbana adulta en la Argentina, se efectuaron regresiones logísticas multinomiales. El supuesto aquí es que los sujetos pueden tener alguna de las siguientes categorías de peso: desnutrición, normopeso, sobrepeso, obesidad tipo 1, obesidad tipo 2 y obesidad tipo 3, calculados en función del Índice de Masa Corporal (IMC), que mide la relación entre peso y altura de los sujetos. En un contexto de regresión esta variable opera como dependiente y debe ser estimada con un modelo logístico de tipo multinomial, debido a que toma más de dos valores posibles $\mathrm{y}$, además, dichos valores no son estrictamente ordenados.

Los modelos multinomiales suelen ser utilizados para predecir la probabilidad de ocurrencia de las distintas categorías de la variable dependiente condicionada al valor de las variables independientes. En este caso, la probabilidad de pertenecer a una de las seis categorías de peso es función de predictores vinculados a factores constitutivos (edad, género), hábitos (consumo de frutas y verduras, tabaquismo, horas diarias de permanencia sentado, grado de intensidad de realización de actividad física, conductas adictivas, como el abuso de bebidas alcohólicas) y socioeconómicos (ingreso, duración de la jornada laboral — que puede incidir en hábitos-y nivel educativo). La selección de las variables explicativas del modelo estimado estuvo guiada por factores que suelen ser mencionados como relevantes en la literatura especializada y condicionada por la disponibilidad de datos publicados por la ENFr. Por ejemplo, entre los hábitos alimentarios que suelen ser valorados en la incidencia de enfermedades no transmisibles se encuentra la ingesta de verduras y frutas y de sal, entre otros (14-16). La ENFR, sin embargo, solo registra si el individuo consume frutas y verduras al menos cinco días a la semana, pero no las cantidades. Algo similar ocurre con el agregado de sal, la ENFR registra la frecuencia (nunca / a veces / casi siempre) con la que el entrevistado agrega sal a las comidas $\mathrm{y}$ dicha intensidad es autoreportada.

Se consideraron, además, variables de control regionales (tabla 2). El término de perturbación en este tipo de modelos se considera aleatorio, independiente y con una distribución de tipo logística (17). 
Tabla 2. Definición de las variables

\begin{tabular}{|c|c|}
\hline Variable & Definición \\
\hline \multicolumn{2}{|c|}{ Categoría de diagnóstico Nutricional } \\
\hline Desnutrición & $\mathrm{IMC} \leq 18,5$ \\
\hline Normopeso & $18,5<\mathrm{IMC} \leq 24,9$ \\
\hline Sobrepeso & $25<\mathrm{IMC} \leq 29,9$ \\
\hline Obesidad T. 1 & $30<\mathrm{IMC} \leq 34,9$ \\
\hline Obesidad T. 2 & $35<\mathrm{IMC} \leq 39,9$ \\
\hline Obesidad T. 3 & IMC $\geq 40$ \\
\hline \multicolumn{2}{|l|}{ Constitutivas } \\
\hline Edad & Edad en años cumplidos \\
\hline Género & $=1$ si mujer; $=0$ en caso contrario \\
\hline \multicolumn{2}{|l|}{ Hábitos } \\
\hline Fumador & $\begin{array}{l}\text { Se considera fumadores actuales a aquellas personas que fuman todos o algunos días en la } \\
\text { actualidad y que a lo largo de su vida han fumado al menos } 100 \text { cigarrillos. }\end{array}$ \\
\hline Hsentado & horas promedio diarias de permanencia sentado \\
\hline Afisintens & $=1 \mathrm{si}$ el individuo reconoce que realiza actividad física intensa $;=0$ en caso contrario \\
\hline \multicolumn{2}{|l|}{ Socioeconómicas } \\
\hline Ingreso & estrato de ingreso familiar mensual, autoreportado \\
\hline Más45h & $=1$ si trabaja 45 horas o más $;=0$ en caso contrario \\
\hline Educpc & $\begin{array}{l}=1 \text { si el nivel de instrucción máximo alcanzado por el sujeto es primaria completa o inferior; } \\
=0 \text { en caso contrario }\end{array}$ \\
\hline \multicolumn{2}{|l|}{ Tipo de Hogar } \\
\hline Hogtrad & $=1$ si hogar multipersonal conyugal completo con hijos; $=0$ en caso contrario \\
\hline Hogsinh & $=1$ si en el hogar no hay hijos menores de edad $;=0$ en caso contrario \\
\hline
\end{tabular}

* Se considera actividad física intensa si el sujeto admite que al menos 3 días a la semana realiza actividad física acumulando 1500 METS-minuto * 07 más días de cualquier combinación de caminata, moderada o intensa acumulando un mínimo de 3000 METS-minuto por semana. MET = razón entre el metabolismo de una persona durante la realización de un trabajo y su metabolismo basal. Se define como el costo energético de estar sentado tranquilamente, es equivalente a un consumo de 1 $\mathrm{kcal} / \mathrm{kg} / \mathrm{h}$

La categoría normal de IMC constituye la base de comparación. La elección de esta categoría responde la facilidad de establecer los signos esperados de los coeficientes que acompañan a los cofactores en el resto de las categorías. Por ende, la probabilidad de que un individuo se encuentre en la categoría base no está determinada en el conjunto presente. Este problema desaparece, de todos modos, al normalizar los coeficientes de la categoría normopeso (18).
La estimación de los coeficientes del modelo se realiza aplicando el método de máxima verosimilitud para el resto de las categorías de IMC. La probabilidad del j-ésimo individuo de pertenecer a la i-ésima categoría de IMC puede ser calculada como (19):

$$
p_{i j}=\frac{e^{\beta k X k j}}{\sum_{k} e^{\beta k X k j}} \quad \operatorname{con} i=1 \ldots m, j=1 \ldots N, k=1 \ldots K
$$


Donde $\mathrm{X}_{\mathrm{kj}}$ representa un conjunto de $k$ predictores correspondientes al individuo j y $\beta_{\mathrm{k}}$ a los parámetros del modelo. La expresión anterior refleja que los modelos logísticos multinomiales pueden ser concebidos como una estimación simultánea de logit binarios para todas las categorías de la dependiente. En este tipo de modelos es crítico evaluar si los resultados se mantienen cuando se elimina alguna de las categorías de la variable dependiente, propiedad conocida como "independencia de las alternativas irrelevantes" (IAI) (20). Por otro lado, como las bases de datos compilan información para una gran cantidad de individuos (46308 observaciones en 2005 y 34934 observaciones en 2009), las propiedades asintóticas de los estimadores se consideran ampliamente cubiertas. No obstante, se debe aclarar que la cantidad de observaciones consideradas en las estimaciones disminuye, respecto del total de personas entrevistadas, ya que no todas han informado la totalidad de respuestas a las preguntas incluidas en los cuestionarios aplicados. La cantidad de observaciones efectiva en los modelos estimados es reportada oportunamente en las tablas de resultados e igualmente es suficiente para asegurar las propiedades de consistencia y eficiencia de los estimadores máximo-verosimiles.

Una ventaja de los modelos logísticos multinomiales es que permiten ser expresados en términos de la razón de riesgo relativo (RRR), que expresa la variación de la probabilidad de pertenecer a la categoría de peso $i$, relativa a la categoría de peso de referencia, en este caso, normopeso, para cada variación unitaria en la variable predictora, dado que todo lo demás permanece constante. Para ilustrar, si se desea evaluar el impacto del género en la desnutrición, un RRR equivalente a 3 implica que ser mujer aumenta en un factor de 3 las chances de padecer desnutrición, con relación a ubicarse en un peso normal, manteniendo el resto de las variables en su nivel. Por otro lado, mientras que RRR $>1$ se interpreta como efecto positivo sobre las chances de pertenecer a la categoría analizada en relación con la de referencia, RRR $<1$ se interpreta en forma inversa; es decir, disminuyen las chances de que el sujeto se ubique en dicha categoría, respecto de la categoría de comparación (normopeso).

El modelo fue estimado con el paquete econométrico Stata 11. Las estimaciones controlaron la existencia de autocorrelación intra-provincia y heterocedasticidad en los errores y se chequearon además diferencias entre estimaciones ponderadas y no ponderadas por la inversa de la probabilidad de selección en el muestreo.

\section{Resultados}

Las tablas 3 y 4 presentan los resultados. Cabe señalar que las variables de hábitos referidas a consumo de frutas y verduras y abuso de bebidas alcohólicas no resultaron significativas en ninguna especificación. Lo mismo ocurrió con la posesión de cobertura de salud, la jefatura femenina del hogar y la residencia en zonas urbanas mayores a 500000 habitantes. Con respecto del cumplimiento del supuesto de IAI, si bien, el signo del estadístico impidió obtener la probabilidad de error tipo I, en algunos casos, en los casos en que el estadístico de contraste resultó significativo no se rechaza la hipótesis nula de independencia de las alternativas irrelevantes, aspecto crítico para continuar con el análisis de los resultados (el detalle de los resultados de los contrastes de Hausman se presenta en el anexo).

Globalmente se observa que la desnutrición depende esencialmente de la edad del sujeto (a medida que aumenta la edad, aumenta el riesgo de desnutrición; al menos para 2005, este efecto sería además exponencial), del género (las mujeres tienen más chances de padecerla) y factores esencialmente 
Tabla 3. Razón de riesgo relativo. Matriz de varianzas y covarianzas estimadas con clustering por provincia para variables constitutivas, hábitos, tipo de hogar y socioeconómicas. 2005

\begin{tabular}{|c|c|c|c|c|c|}
\hline & Desnutrido & Sobrepeso & Obeso 1 & Obeso 2 & Obeso 3 \\
\hline \multicolumn{6}{|l|}{ Constitutivas } \\
\hline Edad & $\begin{array}{c}0,9039 \\
(0,000) \\
{[0,88780,9202]}\end{array}$ & $\begin{array}{c}1,1230 \\
(0,000) \\
{[1,11181,1343]} \\
\end{array}$ & $\begin{array}{c}1,1972 \\
(0,000) \\
{[1,18341,2113]} \\
\end{array}$ & $\begin{array}{c}1,2682 \\
(0,000) \\
{[1,23681,3003]} \\
\end{array}$ & $\begin{array}{c}1,3195 \\
(0,000) \\
{[1,24071,4032]}\end{array}$ \\
\hline Edad2 & $\begin{array}{c}1,0008 \\
(0,000) \\
{[1,00071,001]}\end{array}$ & $\begin{array}{c}0,99908 \\
(0,000) \\
{[0,99900,9992]}\end{array}$ & $\begin{array}{c}0,9985 \\
(0,000) \\
{[0,99840,9986]}\end{array}$ & $\begin{array}{c}0,9979 \\
(0,000) \\
{[0,99770,9981]}\end{array}$ & $\begin{array}{c}0,9974 \\
(0,000) \\
{[0,99680,9981]}\end{array}$ \\
\hline Género & $\begin{array}{c}3,2994 \\
(0,000) \\
{[2,73073,9866]}\end{array}$ & $\begin{array}{c}0,4579 \\
(0,000) \\
{[0,43360,4835]} \\
\end{array}$ & $\begin{array}{c}0,5313 \\
(0,000) \\
{[0,49290,5728]} \\
\end{array}$ & $\begin{array}{c}0,7393^{(b)} \\
(0,001) \\
{[0,62020,8813]} \\
\end{array}$ & $\begin{array}{c}1,09 \\
(0,437)\end{array}$ \\
\hline \multicolumn{6}{|l|}{ Hábitos } \\
\hline Fumador & $\begin{array}{l}1,0936 \\
(, 143)\end{array}$ & $\begin{array}{c}0,8203 \\
(0,000) \\
{[0,77930,8635]} \\
\end{array}$ & $\begin{array}{c}0,7384 \\
(0,000) \\
{[0,68160,7998]} \\
\end{array}$ & $\begin{array}{c}0,6482 \\
(0,000) \\
{[0,57180,7349]} \\
\end{array}$ & $\begin{array}{c}0,6701 \\
(0,013) \\
{[0,4886,919]} \\
\end{array}$ \\
\hline Sentadohs & $\begin{array}{l}0,9945 \\
(0,645)\end{array}$ & $\begin{array}{c}1,015 \\
(0,000) \\
{[1,00671,0235]}\end{array}$ & $\begin{array}{c}1,03336 \\
(0,000) \\
{[1,02311,0442]}\end{array}$ & $\begin{array}{c}1,0635 \\
(0,000) \\
{[1,04741,0799]}\end{array}$ & $\begin{array}{c}1,08 \\
(0,000) \\
{[1,05681,1039]}\end{array}$ \\
\hline Afisintens & $\begin{array}{l}0,9106 \\
(0,297)\end{array}$ & $\begin{array}{c}0,9104^{(\mathrm{b})} \\
(0,014) \\
{[0,84480,9811]} \\
\end{array}$ & $\begin{array}{c}0,7358 \\
(0,001) \\
{[0,61870,875]} \\
\end{array}$ & $\begin{array}{c}0,4129 \\
(0,000) \\
{[0,30510,5588]} \\
\end{array}$ & $\begin{array}{c}0,6723^{(b)} \\
(0,046) \\
{[0,45540,9926]} \\
\end{array}$ \\
\hline \multicolumn{6}{|l|}{ Tipo de hogar } \\
\hline Hogtrad & $\begin{array}{l}0,8651 \\
(0,122)\end{array}$ & $\begin{array}{c}1,1511 \\
(0,000) \\
{[1,07261,02354]}\end{array}$ & $\begin{array}{c}1,1493^{(\mathrm{b})} \\
(0,003) \\
{[1,04681,2618]} \\
\end{array}$ & $\begin{array}{c}1,2076 \\
(0,025) \\
{[1,02391,4242]} \\
\end{array}$ & $\begin{array}{l}1,1683 \\
(0,207)\end{array}$ \\
\hline Hogsinh & $\begin{array}{l}0,9702 \\
(0,733)\end{array}$ & $\begin{array}{c}0,9242 \\
(0,01) \\
{[0,87040,9814]}\end{array}$ & $\begin{array}{c}0,799 \\
(0,000) \\
{[0,71370,8946]}\end{array}$ & $\begin{array}{c}0,7666 \\
(0,019) \\
{[0,61420,9568]}\end{array}$ & $\begin{array}{l}0,9541 \\
(0,732)\end{array}$ \\
\hline \multicolumn{6}{|l|}{ Socioecon } \\
\hline Ingreso & $\begin{array}{c}0,9808^{(\mathrm{a})} \\
(0,088)\end{array}$ & $\begin{array}{c}0,9957^{(\mathrm{c})} \\
(0,305)\end{array}$ & $\begin{array}{l}0,9897 \\
(0,098)\end{array}$ & $\begin{array}{c}0,9752 \\
(0,009) \\
{[0,95690,9938]}\end{array}$ & $\begin{array}{c}0,969 \\
(0,042) \\
{[0,93990,9989]}\end{array}$ \\
\hline Más $45 h$ & $\begin{array}{l}0,9838 \\
(0,84)\end{array}$ & $\begin{array}{c}1,1021 \\
(0,002) \\
{[1,03621,1722]}\end{array}$ & $\begin{array}{c}1,1035 \\
(0,033) \\
{[1,00821,2078]}\end{array}$ & $\begin{array}{l}1,1516 \\
(0,181)\end{array}$ & $\begin{array}{c}1,197 \\
(0,171)\end{array}$ \\
\hline Educpc & $\begin{array}{l}1,0473 \\
(, 635)\end{array}$ & $\begin{array}{c}1,1686 \\
(0,000) \\
{[1,10361,2374]} \\
\end{array}$ & $\begin{array}{c}1,3227 \\
(0,000) \\
{[1,21721,4374]} \\
\end{array}$ & $\begin{array}{c}1,32 \\
(0,002) \\
{[1,10971,5702]}\end{array}$ & $\begin{array}{c}1,2745 \\
(0,02) \\
(1,03981,5622]\end{array}$ \\
\hline $\begin{array}{l}\text { Dummies } \\
\text { regionales }^{(\mathrm{d})}\end{array}$ & $\begin{array}{c}\text { regpamp+* } \\
\text { regnoa+* } \\
\text { regnea+ }^{*} \\
\text { recuyo+ }\end{array}$ & $\begin{array}{l}\text { reggba-* }^{*} \\
\text { regpamp-* } \\
\text { regnea- }^{*}\end{array}$ & $\begin{array}{l}\text { reggba-* } \\
\text { regpamp-* } \\
\text { regnea-* }\end{array}$ & $\begin{array}{l}\text { reggba-** } \\
\text { regpamp-* } \\
\text { regnea-** }\end{array}$ & $\begin{array}{l}\text { reggba-* } \\
\text { regpamp-* } \\
\text { regnea-* } \\
\text { regcuyo-**(b) }\end{array}$ \\
\hline Nro. obs & 34910 & & & & \\
\hline \multicolumn{6}{|c|}{$\begin{array}{l}\text { (a) Significativa al } 5 \% \text { aplicando opción robust } \\
\text { (b) No significativa en regresión ponderada por inversa de probabilidad de selección } \\
\text { (c) Significativa al } 5 \% \text { en estimación ponderada } \\
\text { (d) Se indican solamente las regiones cuyos coeficientes resultaron significativos. } \\
+=\text { efecto positivo, }{ }^{-}=\text {efecto negativo. }{ }^{*}=p \text {-valor }<.01{ }^{* \star} \text { p-valor }<.05 \\
\text { Intervalos de confianza entre corchetes }\end{array}$} \\
\hline te: elaboración & n estimacione & & & & \\
\hline
\end{tabular}


Tabla 4. Razón de riesgo relativo. Matriz de varianzas y covarianzas estimadas con clustering por provincia para variables constitutivas, hábitos, tipo de hogar y socioeconómicas, 2009

\begin{tabular}{|c|c|c|c|c|c|}
\hline & Desnutrido & Sobrepeso & Obeso 1 & Obeso 2 & Obeso 3 \\
\hline \multicolumn{6}{|l|}{ Constitutivas } \\
\hline Edad & $\begin{array}{c}0,953^{(\mathrm{a})} \\
(0,034) \\
{[0,91140,99654]}\end{array}$ & $\begin{array}{c}1,1052 \\
(, 000) \\
{[1,08541,1254]} \\
\end{array}$ & $\begin{array}{c}1,1771 \\
(0,000) \\
{[1,15161,2031]} \\
\end{array}$ & $\begin{array}{c}1,2111 \\
(0,000) \\
{[1,17571,2476]} \\
\end{array}$ & $\begin{array}{c}1,2556 \\
(0,000) \\
{[1,17611,3405]} \\
\end{array}$ \\
\hline Edad2 & $\begin{array}{l}1,0003 \\
(0,279)\end{array}$ & $\begin{array}{c}0,9992 \\
(0,000) \\
{[0,9990,9993]} \\
\end{array}$ & $\begin{array}{c}0,9986 \\
(0,000) \\
{[0, .99840,9988]} \\
\end{array}$ & $\begin{array}{c}0,9983 \\
(0,000) \\
{[0,9980,9985]} \\
\end{array}$ & $\begin{array}{c}0,9978 \\
(0,000) \\
{[0,99710,9985]} \\
\end{array}$ \\
\hline Género & $\begin{array}{c}3,0066 \\
(0,000) \\
{[2,26593,9894]} \\
\end{array}$ & $\begin{array}{c}0, .4628 \\
(0,000) \\
{[0,41830,512]} \\
\end{array}$ & $\begin{array}{c}0, .5333 \\
(0,000) \\
{[0,47630,5972]} \\
\end{array}$ & $\begin{array}{c}0,724^{(\mathrm{e})} \\
(0,001) \\
{[0,59950,8745]} \\
\end{array}$ & $\begin{array}{l}0,8243 \\
(0,073)\end{array}$ \\
\hline \multicolumn{6}{|l|}{ Hábitos } \\
\hline Fumador & $\begin{array}{l}1,1663 \\
(0,353)\end{array}$ & $\begin{array}{c}0,7727 \\
(0,000) \\
{[0,7039,8481]} \\
\end{array}$ & $\begin{array}{c}0,6731 \\
(0,000) \\
{[0,5843,7754]} \\
\end{array}$ & $\begin{array}{c}0,6753 \\
(0,000) \\
{[0,5501,829]} \\
\end{array}$ & $\begin{array}{c}0,4835 \\
(0,000) \\
{[0,3302,7078]} \\
\end{array}$ \\
\hline Sentadohs & $\begin{array}{c}1,006 \\
(0,802)\end{array}$ & $\begin{array}{c}1,0207^{(b)} \\
(0,000) \\
{[1,01051,031]}\end{array}$ & $\begin{array}{c}1,0333 \\
(0,002) \\
{[1,01241,0545]}\end{array}$ & $\begin{array}{c}1,0879 \\
(0,000) \\
{[1,05941,1171]}\end{array}$ & $\begin{array}{c}1,0712 \\
(0,013) \\
{[1,01451,1311]}\end{array}$ \\
\hline Afisintens & $\begin{array}{l}0,9664 \\
(0,829)\end{array}$ & $\begin{array}{c}0,925 \\
(0,287)\end{array}$ & $\begin{array}{c}0,7307 \\
(0,000) \\
{[0,61560,8672]} \\
\end{array}$ & $\begin{array}{c}0,57 \\
(0,000) \\
{[0,43380,749]} \\
\end{array}$ & $\begin{array}{c}0,5193^{(b)} \\
(0,037) \\
{[0,28060,9610]}\end{array}$ \\
\hline \multicolumn{6}{|l|}{ Tipo de hogar } \\
\hline Hogtrad & $\begin{array}{c}0,8495^{(\mathrm{e})} \\
(0,132)\end{array}$ & $\begin{array}{l}1,0674 \\
(0,168)\end{array}$ & $\begin{array}{c}1,2382^{(b)} \\
(0,003) \\
{[1,07311,4287]} \\
\end{array}$ & $\begin{array}{l}1,1356 \\
(0,189)\end{array}$ & $\begin{array}{l}0,9691 \\
(0,861)\end{array}$ \\
\hline Hogsinh & $\begin{array}{l}1,2326 \\
(0,107)\end{array}$ & $\begin{array}{c}0,9137 \\
(0,016) \\
{[0,84910,9832]} \\
\end{array}$ & $\begin{array}{c}0,826 \\
(0,003) \\
{[0,72860,9363]} \\
\end{array}$ & $\begin{array}{c}0,7166 \\
(0,001) \\
{[0,58740,8742]} \\
\end{array}$ & $\begin{array}{c}0,5376 \\
(0,007) \\
{[0,34290,8427]}\end{array}$ \\
\hline \multicolumn{6}{|l|}{ Socioecon } \\
\hline Ingreso & $\begin{array}{c}0,9405 \\
(0,000) \\
{[0,90850,9735]}\end{array}$ & $\begin{array}{l}0,9993 \\
(0,894)\end{array}$ & $\begin{array}{c}0,9997^{(\mathrm{f})} \\
(0,979)\end{array}$ & $\begin{array}{c}0,968 \\
(0,001) \\
{[0,950,9863]}\end{array}$ & $\begin{array}{l}1,0027 \\
(0,888)\end{array}$ \\
\hline Más $45 h$ & $\begin{array}{l}0,8889 \\
(0,509)\end{array}$ & $\begin{array}{c}1,1344 \\
(0,005) \\
{[1,03791,2399]} \\
\end{array}$ & $\begin{array}{c}1,2045 \\
(0,000) \\
{[1,10281,3266]}\end{array}$ & $\begin{array}{c}1,3926 \\
(0,003) \\
{[1,12061,7306]}\end{array}$ & $\begin{array}{c}1,318 \\
(0,088)\end{array}$ \\
\hline Educpc & $\begin{array}{c}1,1343^{(\mathrm{e})} \\
(0,294)\end{array}$ & $\begin{array}{c}1,1027 \\
(0,027) \\
{[1,01131,2025]}\end{array}$ & $\begin{array}{c}1,4518 \\
(0,000) \\
{[1,3211,5955]}\end{array}$ & $\begin{array}{c}1,3367 \\
(0,018) \\
{[1,0511,7]}\end{array}$ & $\begin{array}{c}1,7319 \\
(0,000) \\
{[1,322,2723]}\end{array}$ \\
\hline $\begin{array}{l}\text { Dummies } \\
\text { regionales }\end{array}$ & reggba+** & & & $\begin{array}{l}\text { regpamp-* } \\
\text { regnea-* } \\
\text { regcuyo-*(b) }\end{array}$ & $\begin{array}{l}\text { regpamp-** } \\
\text { regcuyo- }^{* *}\end{array}$ \\
\hline Nro. obs & 15949 & & & & \\
\hline
\end{tabular}

(a) Significativa al $5 \%$ aplicando opción robust

(b) № significativa en regresión ponderada por inversa de probabilidad de selección

(c) Significativa al $5 \%$ en estimación ponderada

(d) Se indican solamente las regiones cuyos coeficientes resultaron significativos

$+=$ efecto positivo, ${ }^{-}=$efecto negativo. ${ }^{*}=$ p-valor $<.01{ }^{* \star}$ p-valor $<.05$

(e) Significativa al $5 \%$ en estimación ponderada

(f) Significativa al $1 \%$ en estimación ponderada

Intervalos de confianza entre corchetes

Fuente: elaboración basado en estimaciones. 
socioeconómicos (como el nivel de ingreso, que resulta significativo en 2009 o las variables indicadoras de la región de residencia que captan especificidades del entorno socioeconómico). No hay evidencia de que los hábitos o tipo de hogar sean significativos en la desnutrición.

La escasez de predictores de desnutrición puede deberse a dos factores: por un lado, la desnutrición aquí captada se apoya únicamente en la relación peso/talla, mientras que la problemática comprende otros aspectos que no necesariamente son adecuadamente captados por este indicador, como el déficit de proteínas. Así, es posible que un individuo se encuentre desnutrido, aunque sus parámetros de peso para la talla se ubiquen en valores normales. El segundo elemento que puede estar influyendo en los resultados responde al tipo de variables de hábitos registradas en la ENFR, más enfocada en la ingesta de sustancias saludables y/o recomendadas para reducir fenómenos como colesterolemia, accidentes cardiovasculares, etcétera, pero no captura hábitos alimentarios vinculados propiamente a la ingesta de nutrientes. Con todo, se verifica que la desnutrición aumenta en edades avanzadas, afecta en mayor medida a las mujeres y está vinculada a cuestiones socioeconómicas.

Respecto de los problemas de sobrepeso y obesidad, las estimaciones muestran que la edad incide en forma opuesta que la observada en desnutrición. Si bien, la edad aumenta los riesgos de padecer problemas de peso, ello ocurre a un ritmo decreciente. Por otro lado, el efecto de la edad sobre el riesgo relativo es creciente en las categorías de obesidad (grado 1, 2 y 3).

Por su parte, las mujeres tienen menor riesgo relativo de padecer exceso de peso, especialmente en las categorías de sobrepeso y obesidad tipo 1. A medida que se avanza en las categorías de obesidad, la desventaja de los varones respecto de las mujeres decrece y se vuelve nula en la obesidad mórbida.
El impacto del tabaquismo sobre el exceso de peso es significativo. Los sujetos fumadores tienen menos chances relativas de padecer problemas de peso. El impacto negativo es creciente en las categorías de sobrepeso, fumar reduce más el riesgo relativo de obesidad tipo 3 que el de sobrepeso.

La cantidad de horas diarias que el sujeto permanece sentado incide positivamente en el exceso de peso con un efecto levemente creciente a medida que se avanza en las categorías. Con todo, la magnitud del impacto es sustancialmente menor a otros predictores.

La realización de actividad física intensa por parte del individuo reduce el riesgo relativo en las categorías intermedias de obesidad (tipo 1 y 2). En las situaciones extremas de exceso de peso (sobrepeso y obesidad mórbida), la actividad física intensa reduce el RRR de padecer sobrepeso y obesidad mórbida, aunque la amplitud del intervalo de confianza se amplía indicando más incertidumbre sobre su impacto.

Por su parte, la pertenencia a un hogar tradicional (conformado por pareja conyugal e hijos) no resulta robusta en las estimaciones, mientras que en 2005 aumentaba el riesgo relativo de experimentar exceso de peso, en 2009 este efecto solo resulta significativo en la obesidad tipo 1. Los sujetos que viven en hogares sin niños tienen menos chances de padecer exceso de peso. La magnitud del impacto es mayor a medida que la obesidad avanza en grado.

Los factores socioeconómicos tienen una incidencia significativa en los problemas de peso. Esto se manifiesta, especialmente, si se toma a la educación del sujeto como proxy del nivel socioeconómico. Bajos niveles de instrucción están positivamente asociados con un mayor riesgo relativo de problemas de peso. Por su parte, los individuos expuestos a jornadas laborales extensas tienen mayores RRR de experimentar exceso de peso. Este efecto es 
particularmente robusto en el sobrepeso y obesidad tipo 1. El nivel de ingreso parece reducir el riesgo relativo de problemas alimentarios, aunque las estimaciones muestran ausencia de robustez. En 2005 el nivel de ingreso es significativo en la reducción del riesgo relativo de la obesidad tipo 2 y 3, aunque en 2009 el ingreso solo resultó relevante en la reducción de la obesidad tipo 2. La falta de robustez en la variable puede originarse en 2 fenómenos: por un lado, la estrecha correlación entre el ingreso y el resto de las variables socioeconómicas (especialmente, con la educación del jefe de hogar), que tiende a inflar la varianza de los estimadores y a anular la significatividad de alguno de los regresores correlacionados, en este caso, el ingreso. Por otro lado, dado que se trata de un relevamiento autoreportado, es común que los respondentes tiendan a subdeclarar ingresos, lo que disminuye la varianza del predictor $\mathrm{y}$ posiblemente su significatividad individual.

\section{Discusión}

Los cambios en los perfiles demográficos, epidemiológicos y sanitarios acaecidos en las últimas décadas han dado como resultado la aparición de nuevos problemas nutricionales, relacionados con el exceso de consumo de alimentos en general o de aquellos con bajos aportes nutricionales y excedente de calorías. Esta situación se traduce en una expansión significativa del sobrepeso y la obesidad que, a su vez, se encuentran relacionadas con un amplio abanico de enfermedades crónicas en aumento. Las causas de esta epidemia son muchas y variadas: la disminución del esfuerzo físico en el trabajo y en el hogar, la relación de precios entre alimentos saludables y no saludables, la incorporación de la mujer en el mercado laboral, las formas de recreación cada vez más sedentarias, la diagramación de las ciudades o la inseguridad urbana que desincentivan desplazarse caminando, son algunas de las más citadas.
Simultáneamente, la desnutrición continúa presente. Por primera vez en la historia, existen en el mundo tantos individuos desnutridos como obesos.

La problemática nutricional en la Argentina es compleja. Al igual que en el resto de América Latina, las desigualdades en el ingreso son marcadas (20). Existen regiones con elevados y persistentes niveles de pobreza estructural, con limitaciones en el acceso a los alimentos en general o a alimentos nutricionalmente aceptables. Consecuencia de esto, las tasas de desnutrición muestran resistencia a la baja, aun cuando la Argentina se caracteriza por su amplia producción de alimentos. Por otro lado, y en forma simultánea, los problemas relacionados con el exceso de peso se incrementan aceleradamente.

Los grupos de mayores ingresos suelen exhibir una dieta basada en elevados niveles de consumo de proteínas, grasas, hidratos de carbono y azúcares simples. Por su parte, en los de ingresos más bajos predomina el consumo de cereales, azúcares simples y fibras. Ambas dietas, si bien diferentes, son nutricionalmente deficientes. El primer grupo puede manifestar patologías relacionadas con la obesidad y el consumo excesivo de grasas, mientras que en el segundo es frecuente la desnutrición simultánea a la obesidad con carencias de nutrientes esenciales.

Del análisis aquí presentado, surge que en la Argentina la desnutrición se asocia con las variables constitutivas y socioeconómicas, mientras que en la obesidad intervienen además los hábitos.

Existe una estrecha vinculación entre la desnutrición en adultos y las condiciones socioeconómicas. A su vez, la prevalencia de la enfermedad es mayor en las mujeres y aumenta con la edad, situación que acentúa la vulnerabilidad en la vejez.

En el caso de la obesidad, los resultados son más complejos. Dado que el incremento de peso se da por un desbalance entre el consumo calórico 
y el gasto energético, una variedad de factores pueden influir sobre este. Asimismo, la importancia relativa de estas causas es diferente entre regiones, por lo que el conocimiento de estas situaciones es fundamental para la implementación de políticas adecuadas.

Si bien, las condiciones socioeconómicas están estrechamente relacionadas con el exceso de peso, en especial la educación, los factores constitutivos del individuo, los hábitos y el tipo de hogar en el que se habita han mostrado influir sobre las probabilidades de padecer la enfermedad.

Entre los hábitos considerados, es llamativa la relación inversa entre tabaquismo y obesidad. Como esta relación tiene implicancias paradójicas en términos de resultados deseables de posibles intervenciones públicas (e.g. campañas antitabaco no serían neutrales frente a la obesidad), se destaca la necesidad de un análisis más profundo que tome en cuenta este resultado. Una ruta posible de análisis podría estar enfocada en la teoría de las adicciones.

Por otro lado, el hecho de que los datos aquí empleados sean autoreportados y otros carez- can de detalle suficiente (por ejemplo, la ingesta de sal, frutas y verduras, aceites, etcétera) puede introducir cierta ineficiencia en las estimaciones. Ello destaca la necesidad de utilizar fuentes de información complementaria que añadan riqueza a la información y permitan mejorar la precisión de los resultados.

La estrecha relación entre los problemas nutricionales, las condiciones socioeconómicas y los hábitos pone de relieve la importancia del rol de políticas sanitarias preventivas, tendientes a concientizar sobre hábitos alimentarios e intervenciones sociales que mejoren las condiciones socioeconómicas de las personas e incidan, por ende, en sus oportunidades alimentarias.

Dada la magnitud de los problemas nutricionales, en especial el avance del exceso de peso como problema central de salud pública, su abordaje es de vital importancia para mejorar la calidad de vida de los sujetos, aliviar las presiones sobre los sistemas sanitarios y, fundamentalmente, favorecer el crecimiento y desarrollo del país.

\section{Referencias}

1. Komlos J, Breitfelder A, Sunder M. The transition to post-industrial BMI values among US children. Am J Hum Biol. 2008; 21:151-60.

2. Peña M, Bacallao J. La obesidad y sus tendencias en la región. Rev Pan Salud Púb. 2001;10:75-8.

3. London S, Temporelli K, Monterubbianesi P. Vinculación entre salud, ingreso y educación: Un análisis comparativo para América Latina. Economía y Sociedad 2009;14:125-46.

4. Ogden C, Carroll M, Curtin LR, McDowell MA, Tabak CJ, Flegal KM. Prevalence of overweight and obesity in the United States, 1999-2004. J Am Med Ass. 2006;295(6):1549-55.

5. Jolliffe D. Overweight and poor? On the relationship between income and the body mass index. Econ Hum Biol 2006;9:342-55.

6. Do DP, Dubowitz T, Bird, C, Lurie N, Escarce JJ, Finch B. Neighborhood context and ethnicity differences in body mass index: A multilevel analysis using the NHANES III survey (1988-1994). Econ Hum Biol. 2007;6:179-203.

7. Zhang $Q$, Wang Y. Socioeconomic inequality of obesity in the United States: do gender, age, and ethnicity matter? Social Sci Med 2004;58:1171-80. 
8. Amador M. Seminario Taller sobre obesidad y pobreza en América Latina. Revista Cubana Alimentación y Nutrición [internet]. 1996;10(1) [citado 2013 ago 5]. Disponible en: http://bvs.sld.cu/revistas/ ali/vol10_1_96/ali13196.htm

9. Caro AR, López-Valcárcel BG. El trasfondo económico de las intervenciones sanitarias en la prevención de la obesidad. Rev Esp Salud Pública 2009;83(1),25-41.

10. Serrano RM. Metabolic syndrome: a modern variant of stress-related disease? Rev Esp Cardio. 2005;58:768-71.

11. Lakdawalla D, Philipson T. The Growth of Obesity and Technological Change: A Theoretical and Empirical Investigation. National Bureau of Economic Research. Working Paper No. 8965 [internet]. 2002 May [citado 2013 jul 5]. Disponible en: http://www.nber.org/papers/w8946

12. Temporelli K, Viego V. Obesidad, sobrepeso y condiciones socioeconómicas. El caso argentino. Ecos de Economía. 2012;16(34):151-62.

13. Viego V, Temporelli K. Obesidad y Sobrepeso en Argentina. Una aplicación de Econometría Espacial. Estudios de Economía Aplicada. 2011;29(3):1-26.

14. Joffe $\mathrm{M}$, Robertson A. The potential contribution of increased vegetable and fruit consumption to health gain in the European union. Public Health Nutr. 2001;4(4): 893-901.

15. Trichopoulou A, Naska A, Antoniou A, Friel S, Trygg K, Turrini A. Vegetable and fruit: the evidence in their favour and the public health perspective. Int J Vitam Nutr Res. 2003;73(2):63-9.

16. He F, MacGregor G. Reducing Population Salt Intake Worldwide: From Evidence to Implementation. Prog Cardio Dis. 2010;52(5):363-82.

17. Long S, Freese J. Regression models for categorical dependent variables using Stata. College Station, Texas: Stata Press; 2006.

18. Greene W. Econometric Analysis 5. a ed. New Jersey: Pearson Education; 2003.

19. Small K, Hsiao C. Multinomial logit specification tests. Int Ec ver. 1958;26:619-27.

20. Doris-Cardona L, Acosta D, Bertone CL. Inequidades en salud entre países de Latinoamérica y el Caribe (2005-2010). Gac Sanit. 2013;27:292-7. 